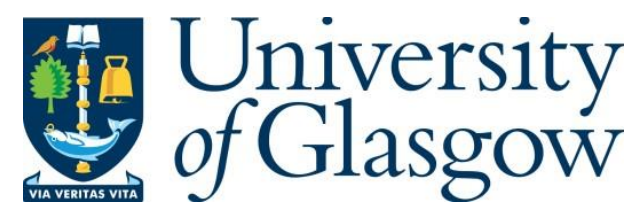

You, Z., Zahid, A., Heidari, H., Imran, M. and Abbasi, Q. H. (2019) A Compact

Wearable System for Detection of Plantar Pressure for Diabetic Foot Prevention. In: IEEE Asia Pacific Conference on Postgraduate Research in Microelectronics and Electronics (PrimeAsia 2018), Chengdu, China, 26-30 Oct 2018, pp. 64-67. ISBN 9781538695913.

There may be differences between this version and the published version. You are advised to consult the publisher's version if you wish to cite from it.

http://eprints.gla.ac.uk/168792/

Deposited on: 13 September 2018

Enlighten - Research publications by members of the University of Glasgow http://eprints.gla.ac.uk 


\title{
A Compact Wearable System for Detection of Plantar Pressure for Diabetic Foot Prevention
}

\author{
Zihang You ${ }^{1}$, Adnan Zahid ${ }^{2}$, Hadi Heidari², Muhammad Imran'2, Qammer H. Abbasi ${ }^{2}$ \\ ${ }^{1}$ University of Electronic Science and Technology of China, Chengdu, China \\ ${ }^{2}$ School Electronics and Nanoscale Engineering, University of Glasgow, G12 8QQ, Glasgow, UK \\ Email: 2168707Y@student.gla.ac.uk, a.zahid.1@research.gla.ac.uk,Hadi.Heidari@glasgow.ac.uk, \\ Muhammad.Imran@glasgow.ac.uk, Qammer.Abbasi@glasgow.ac.uk
}

\begin{abstract}
This paper is aimed to present a pair of wearable shoe-pads with shoes to detect the pressure and the variation of plantar pressure of human body and provide the pressure map with an alarm to the patient. In this work, a complete system is developed which includes data collection circuit, wireless transmission circuit and data analysis. Pressure sensors are employed to collect the data of plantar pressure, send analogue signals to the microcontroller, and a Bluetooth module transmits the data to a PC. Finally, the data is examined and display a hot map of plantar pressure. In addition, by observing the color patterns on a hot map, user or medical staff can judge whether the patient has tendency to develop an ulcer in case of diabetic patient
\end{abstract}

Index Terms: plantar pressure, wearable, pressure sensors

\section{INTRODUCTION}

Diabetes have been a major chronic disease in China. The morbidity of diabetes among residents above 18 years old was $9.7 \%$ in 2010. Among diabetics who have known they have diabetes themselves and received treatments, the glucose control level was $34.7 \%$. Furthermore. a serious problem linked to diabetes is that patients tend to develop foot ulcers. $15 \% \sim 20 \%$ of people with diabetes develop ulcers while $15 \%$ of ulcers result in amputations, and 14\% 24\% of the patients usually need amputation.[1] By detecting the plantar pressure, the symptom of diabetic foot can be predicted and prevented, because the increasing of plantar pressure is the independent risk factor of diabetic foot and the correlation between the increasing of plantar pressure and diabetic foot is up to 70\% 90\%.[2] An effective method to prevent foot ulcers is to check the feet to make sure that there are no wounds, oedemata or blisters. In fact, all of these conditions can be reflected to the feeling of feet, which is directly related to the pressure of the sole of the feet. Pressure sensors can detect the pressure from feet and transmit data to a terminal equipment. Actually, pelma pressure detecting is a relatively mature technology in some countries. For example, F-Scan system from the U.S., Pedar-X measurement system from Germany, and tablet system from Rsscan, a Belgian company, are leading solutions over the world. All of these systems have high-density sensing units and high sample frequency $(100 \sim 300 \mathrm{~Hz})$ [3][4].

For this design, the FSR402 pressure sensors are used to detect the force added on the shoe-pads. Then the microcontroller collects the data and transmit them through Bluetooth modules. Then the data are processed on MATLAB and all the pressure data will be displayed as a hot map which will be refreshed in real time. Most of the known devices used to measure human body's plantar pressure are desktops. Therefore the hot map they output are static. Besides, desktop type devices are not portable. With 2 microcontrollers and 2 Bluetooth modules in 2 shoe-pads, this device are wearable and can transmit pressure data independently. The hot map is also refreshed in real time.

\section{PRESSURE DECTION AREA}

\section{A. The placement of the pressure sensors}

16 pressure sensors are considered for the purpose of collecting the pressure data, and were placed at the places where can be easily detected. For normal people, the distribution of the pressure of pelma is the same when people are walking and standing. The distribution of pressure of 2 soles sustained is symmetrical. There are no significant differences of distribution among males and females. Each part of a sole to sustain the pressure when people walking ranks as followed: the second metatarsal head, the heel, the first metatarsal head, the third to the fifth metatarsal head, the first toe, the second toe, the arch, and the third to the fifth toe. Each part of a foot to sustain the pressure when people standing ranks as followed: the heel, the second metatarsal head, the first metatarsal head, the first 3 to 5 metatarsal head, the arch, the first toe, the second toe, and the third to the fifth toe [3][5].

The placement of the sensors should cover the places where the region of the pelma sustain the most pressure. The five regions with higher plantar pressure were the middle of foot, the outer side of heel, the inner side of heel, the second metatarsal and the first phalange [6]. In addition, the peak pressure of the third metatarsal is also significantly increased under certain pathological conditions. A general distribution of the pressure sensors is shown in Fig 1. 


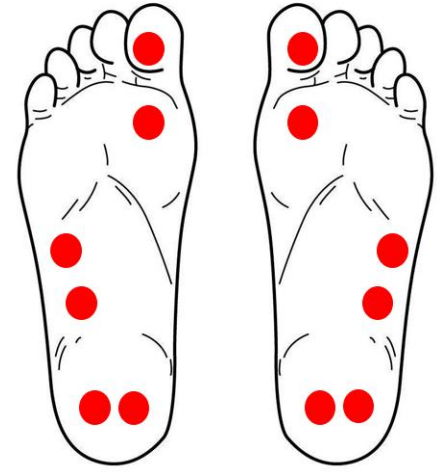

Fig 1. A general distribution of the pressure sensors (red dots) that cover the most sensitive regions of soles

\section{HARDWARE DESIGN}

\section{A. Data Collecting Circuit Design}

The pressure sensor chosen for this design is FSR402. This type of sensor is constructed by polymer film. The length of FSR402 is $53 \mathrm{~mm}$, the diameter of the sensitive area is $7.6 \mathrm{~mm}$, and the thickness is $0.3 \mathrm{~mm}$. The diameter of the circular active area of the sensor is 0.5 inch [7]. It is slim, gentle, and small. Therefore, it is suitable for the detection of plantar pressure. As the force added on the active area increases, the resistance of the FSR decreases. The relation curve between resistance and force satisfies an exponential function. The most basic circuit for pressure signal collecting is to connect FSR402 with a resistor in series to make up a basic voltage divider. In this design, each pressure sensor is connected to a $10 \mathrm{k} \Omega$ resistor. The relationship between the resistance of FSR402 and force is shown in Fig 2.[7] According to Fig 2, when there is no force added on the active area, the resistance of FSR402 is over $100 \mathrm{k} \Omega$, which is much larger than the $10 \mathrm{k} \Omega$ resistance. The output voltage can be recognized as the same with the input voltage. When the force add on the active area is over $10 \mathrm{~kg}$, the resistance of FSR402 is close to $0.1 \mathrm{k} \Omega$, which is much smaller than the $10 \mathrm{k} \Omega$ resistance. The output voltage can be recognized as $0 \mathrm{~V}$.

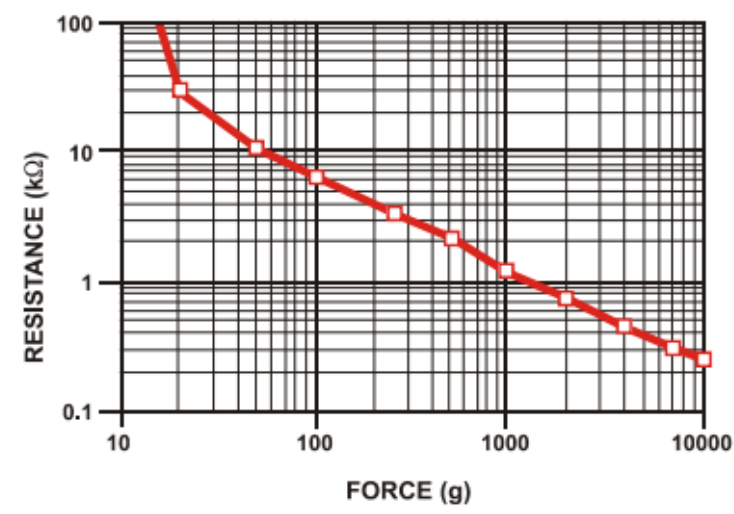

Fig 2. The relationship between the resistance of FSR402 and force

In order to input all signals from 16 pressure sensors, an analog multiplexer CD74HC4067 is used. CD74HC4067 is a 16- channel digitally controlled analog multiplexer developed by Texas Instruments. The multiplexer scans each channel and transmits the analog signal to pin 20 of the LPC1768.

\section{B. Wireless Transmission}

The LPC1768 receives the analog signal and converts it to digital signal. The Bluetooth will transmit a character string with 16 data in it. In this design, the method of wireless transmission is to use 2 HC-05 Bluetooth modules for each shoe-pad. The baud rate is 9600,8 words long and no stop bits. One of the modules acting as a slave is connected to pin 9 and pin 10 of LPC1768, and the transmission method between them is UART. Another module acting as a master is connected with PC by using a TTL to USB module.

\section{Power Supply Circuit}

The schematic diagram of the power supply circuit is shown in Fig 3. The Bluetooth module HC-05 and LPC1768 need the $5 \mathrm{~V}$ power supply. The multiplexer CD74HC4067 and pressure sensors FSR402 need the $3.3 \mathrm{~V}$ power supply. The main power is a $9 \mathrm{~V}$ battery. With 2 voltage regulators L78S05 and LM11173.3 , the $5 \mathrm{~V}$ voltage and $3.3 \mathrm{~V}$ voltage can be generated.

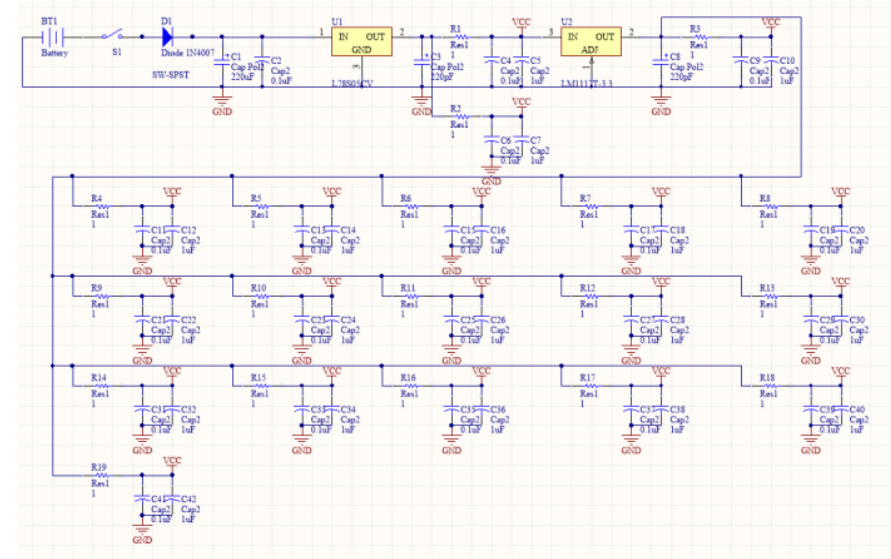

Fig 3. The schematic diagram of the power supply circuit

The input voltage to drive LPC1768 is $4.5 \sim 9.0 \mathrm{~V}$, and to drive $\mathrm{HC}-05$ is $3.6 \sim 6 \mathrm{~V}$. The voltage regulator $\mathrm{L} 78 \mathrm{~S} 05$ has DC input voltage $5 \sim 18 \mathrm{~V}$, and its output voltage is $5 \mathrm{~V}$.[8] The input voltage of LM1117-3.3 is 5V, which fits the output of L78S05. Since the output current of L78S05 is internally limited, the whole circuit will not overheat. For the output of LM1117-3.3, all the pressure sensors and the multiplexer have the input voltage of $3.3 \mathrm{~V}$, which fits the output of LM1117-3.3 [9]. The standard output current for L78S05 is $500 \mathrm{~mA}$, and the maximum output current is internally limited, the output current will not bring troubles to the LTC1768 and HC-05, of which both have overcurrent protection [8]. The input current for the multiplexer CD74HC4067 is 1mA. For the maximum current passing through a pressure sensor FSR402, the current reaches the maximum when the resistance of the pressure sensor has the minimum, and the total resistance of this circuit can be recognised as $10 \mathrm{k} \Omega$. Therefore, the maximum current passing through a pressure sensor is $0.33 \mathrm{~mA}$, and the total output current for LM1117-3.3 is $5.38 \mathrm{~mA}$ (16 parallel branches and the multiplexer).[9] Since when the output voltage is $3.3 \mathrm{~V}$, the 
output current is $10 \mathrm{~mA}$, it is enough for LM1117-3.3 to power all the pressure sensors and the multiplexer.

In Fig 3, in order to prevent the input power connected reversely, and to ensure the current is transmitted unidirectionally, a rectifier diode 1N4007 is connected after the switch. All the electrolytic capacitors and bypass capacitors are used to filter the alternating component with different frequency of the input power supply. For all the branches connecting the microcontroller, modules and sensors, there are $191 \Omega$ resistors connecting in each branch. These resistors have the function to isolate the power supply of each branch and prevent the interference of the noise, because a $1 \mathrm{ohm}$ resistor has a function of being a weak inductance and a fuse.

\section{DATA PRocessing}

\section{A. Data Processing in LPC1768}

The mbed LPC1768 is produced by NXP. It is based on the NXP LPC1768, with a 32-bit ARM Cortex-M3 core running at 96MHz. Mbed LPC1768 has a 12-bit ADC inside, and the reference voltage of this ADC is 3.3V.[10] Therefore, the output voltage as well as the input voltage for all the pressure sensors should be $3.3 \mathrm{~V}$. According to the Equation (1), where $V_{i}$ is the input voltage, $V_{r}$ is the reference voltage, $n$ is the number of bits in the converter output, and $D$ is the digital output value, the equation for calculating the step can be derived. This equation is shown in Equation (2).

$$
\begin{gathered}
D=\frac{V_{i}}{V_{r}} \times 2^{n}[10] \\
\text { step }=\frac{V_{r}}{2^{n}}
\end{gathered}
$$

According to Equation (2), the step of the ADC in LPC1768 is $0.8 \mathrm{mV}$.

For the output of the ADC inside mbed LPC1768, it has 2 forms: unsigned binary form and floating numbers. The digital output range in shape of floating numbers is from 0 to 1 , and the precision can reach to four decimal places. In order to display the correct voltage value, the output floating numbers should be multiplied by 3.3 .

\section{B. Data Processing on MATLAB}

After MATLAB receiving the voltage data, a mathematical model should be constructed to find out the relationship between weight and voltage. According to Fig 2, a relation curve between the resistance and the force is given, but the corresponding function is not given. For this part, a set of M1 level weights is used to demarcate. Since each weight has different size, a piece of clay is used to be as a pedestal for the weights to ensure all the weights have the same base area. When the clay is put on FSR402, the output value on the serial monitor is 0 and the value is stable. This phenomenon indicates that the weight of the clay does not affect the measurement. The process of demarcating is as followed:

1) Place the column on the center of active area of FSR402, and place a weight on it. The center of active area, the clay and the weight should be on the same line.

2) Run the program and do analog-digital conversion. Then Write down the measured value.

The weights used in this experiment is $20 \mathrm{~g}, 50 \mathrm{~g}, 100 \mathrm{~g}, 200 \mathrm{~g}$, $500 \mathrm{~g}$, and $1000 \mathrm{~g}$. The output values and corresponding weights are shown in Table 1.

TABLE1. The output values from ADC and corresponding weights

\begin{tabular}{cc}
\hline \hline Output Value & Weights (Force) /g \\
\hline 0.018 & 20 \\
0.318 & 50 \\
0.618 & 100 \\
0.738 & 200 \\
0.844 & 500 \\
0.925 & 1000 \\
\hline \hline
\end{tabular}

By using the curve fitting application in MATLAB, the scatter diagram and the corresponding fitted curve is shown in Fig 4.

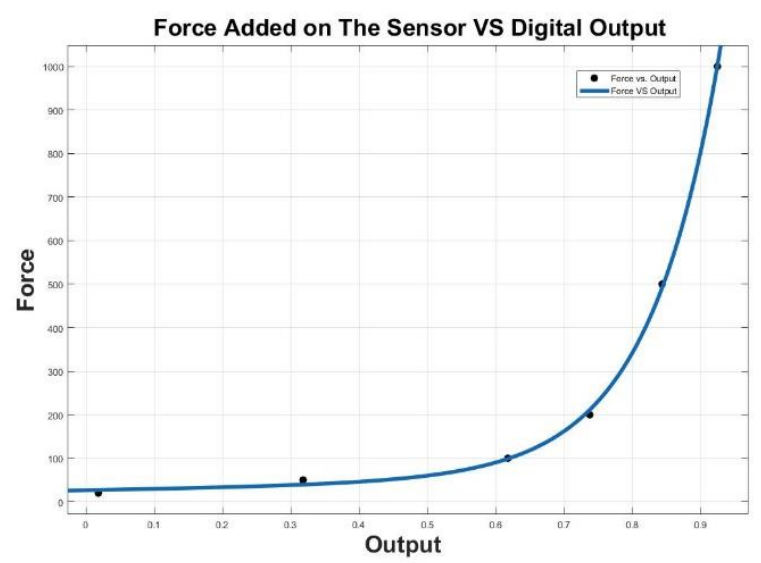

Fig 4. The scatter diagram and the corresponding fitted curve

The equation of the fitted curve is shown in Equation (3), where $F$ corresponds to the force and $x$ corresponds to the output value.

$$
\mathrm{F}=0.128 \times e^{9.615 x}+26.51 \times e^{1.03 x}
$$

\section{Draw the hot map on MATLAB}

Since the hot map is derived from matrix, the first thing is to change the format of data. The receiving data on MATLAB from the Bluetooth module is character string. The first step is to extract the data in the character string, and then arrange them into a matrix in order to build the hot map. In order to display a profile of feet, the matrix should be enlarged, each receiving datum should be rearranged, and in order to display the profile, each datum should be copied to nearby elements. Each datum represents a patch in the hot map, and then one patch is enlarged to a polygonal patch after each datum is enlarged. The final distribution of the hot map is shown in Fig 5. 


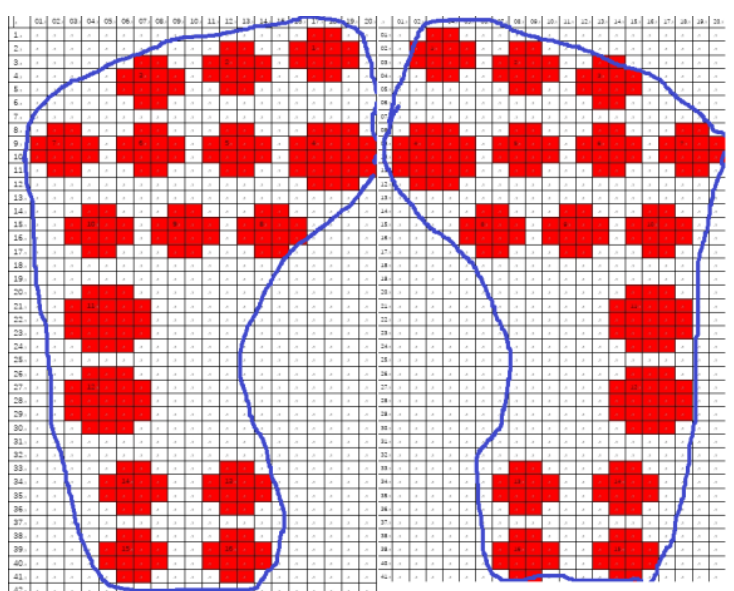

Fig 5. The distribution of the patches

According to Fig 5, the scale of the matrix set for each planta is $41 \times 20$, and all the sensitive areas on planta are covered. An example result of the hot map is shown in Fig 6.
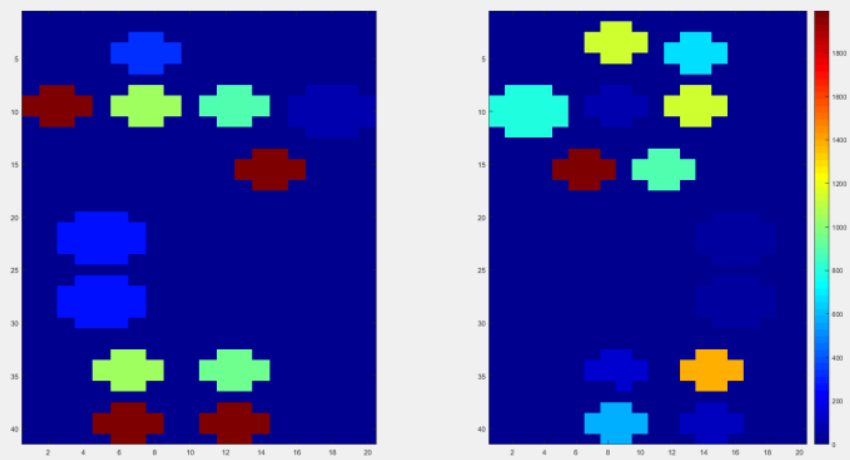

Fig 6. An example of the hot map

\section{CONCLUSION}

The design of the wearable plantar pressure detecting shoepads introduced in this article has achieved the original goals set. The user of this device can visualize the hot map on the screen, which displays the general profile of planta. When the user is walking, the hot map will be refreshed, showing the variation of the plantar pressure. The distribution of the pressure sensors has covered the most sensitive areas of planta. Therefore, the hot map reflects the reliable and scientific data. The purpose of data is to help users to understand the conditions of their feet. Furthermore, wireless Bluetooth transmission helps the device to become wearable and provides an opportunity for the device to offer a real-time hot map to users.

\section{REFERENCES}

[1] Chinese Center For Disease Control And Prevention, "Diabetes and Control," in Report on Chronic Disease Risk Factor Survellance in China 2010, in Military Medical Science Press, 1st ed. Beijing, 2012, pp. 62-65

[2] L A. Lavery, D G. Armstrong, R P. Wunderlich, J. Tredwell, et al. Predictive Value of Foot Pressure Assessment as Part of a Populationbased Diabetes Disease Management Program[J]. Diabetes Care, 2003, 26(4): 1069-73.

[3] Gang Yuan, Muxun Zhang, Zhongqin, Wang Zhongqin, Jianhua Zhang, "The distribution of foot pressure and its influence factors in Chinese people", Chin J Phys Med Rehabil, Vol.26, No.3, pp. 156-159, March 2004

[4] Yunbo Zhang, "A Design of Wearable And Insole Plantar Pressure Measurement System Based On The Stm32," M.S. thesis, School of Life Science and Technology, UESTC, Chengdu, 2016

[5] Deng Xiang, Dian Songyi, Weng Tao, "Wearable plantar pressure detecting system based on FSR", Transducer and Microsystem Technologies, Vol.32, No.2, pp. 81-83.

[6] Qing Yang, "A Design of The Plantar Pressure Measurement Device Based on Embedded System," M.S. thesis, School of Life Science and Technology, UESTC, Chengdu, 2010

[7] Interlink Electronics staff, Force Sensing Resistor Integration Guide and Evaluation Parts Catalog, Interlink Electronics

[8] SGS-THOMSON Microelectronics staff, L78S00 Series 2A Positive Voltage Regulators, SGS-THOMSON Microelectronics, 1994

[9] National Semiconductor Staff, LM1117/LM1117I 800mA Low-Dropout Linear Regulator General Description, National Semiconductor, 2004

[10] R. Toulson, T. Wilmshurst, Fast and Effective Embedded Systems Design Applying the ARM mbed, 2012 